\title{
Von-Neumann Finiteness and Reversibility in some Classes of Non-Associative Algebras
}

\section{Erik Darpö ${ }^{1}$ • Patrik Nystedt ${ }^{2}$ (D)}

Received: 8 May 2018 / Accepted: 11 August 2020 / Published online: 29 August 2020

(C) The Author(s) 2020

\begin{abstract}
We investigate criteria for von-Neumann finiteness and reversibility in some classes of non-associative algebras. Types of algebras that are studied include alternative, flexible, quadratic and involutive algebras, as well as algebras obtained by the Cayley-Dickson doubling process. Our results include precise criteria for von-Neumann finiteness and reversibility of involutive algebras in terms of isomorphism types of their 3-dimensional subalgebras.
\end{abstract}

Keywords Von-Neumann finite $\cdot$ Dedekind finite $\cdot$ Directly finite $\cdot$ Reversible $\cdot$ Quadratic algebra $\cdot$ Involutive algebra

Mathematics Subject Classification (2010) 17D05 - 16K20 - 17C55 · 16W10 - 16U99

\section{Introduction}

A unital ring $A$ is called von-Neumann finite (or Dedekind finite, or weakly 1-finite, or affine finite, or directly finite, or inverse symmetric) if every one-sided inverse in $A$ also is two-sided, in other words, if for all $a, b \in A$ satisfying $a b=1$, the relation $b a=1$ also holds. Many different classes of associative rings have been shown to be vonNeumann finite, for instance noetherian, self-injective and PI-rings [13]. Also group rings over fields of characteristic zero [10, 18, 20], or, more generally, endomorphism rings of permutation modules over such rings $[15,16]$, have been shown to be von-Neumann finite. Von-Neumann finiteness for group rings of positive characteristic has, however, remained

Presented by: Steffen Koenig

Patrik Nystedt

patrik.nystedt@hv.se

Erik Darpö

darpo@math.nagoya-u.ac.jp

1 Graduate School of Mathematics, Nagoya University, Furo-cho, Chikusa-ku, Nagoya, 464-8602, Japan

2 Department of Engineering Science, University West, SE-46186 Trollhättan, Sweden 
an open problem (for partial results, see [3] and [9]). Of course, not all associative unital rings are von-Neumann finite; for instance if $V$ is a vector space, then it is easy to see that $\operatorname{End}(V)$ is von-Neumann finite if and only if $V$ is finite dimensional.

Following Cohn [4], we say that $A$ is reversible if for all $a, b \in A$ satisfying $a b=0$, the relation $b a=0$ also holds. It is easy to see that the class of associative reversible rings is properly contained in the class of associative von-Neumann finite rings. Indeed, suppose that $A$ is an associative reversible ring and $a b=1$ for some $a, b \in A$. Then, since $(b a-1) b=0$, we get that $b(b a-1)=0$ that is $b^{2} a=b$ which implies that $b a=a b b a=a b=1$. Moreover, if $V$ is a vector space satisfying $1<\operatorname{dim}(V)<\infty$, then $\operatorname{End}(V)$ is von-Neumann finite but not reversible.

In this article, we consider von-Neumann finiteness and reversibility for some classes of unital rings which are not associative - apparently a new line of investigation. Our motivation for doing this is two-fold. First, we wish to see to which extent patterns and phenomena from the associative context reproduce in more general classes of algebras, and how far the associative theory can be generalised. Second, the notions of von-Neumann finiteness and reversibility are relevant by themselves in the structure theory of non-associative algebras. Particularly, a long-standing problem has been to understand the structure of quadratic division algebras (see, e.g., [5-8, 14, 19]). The division property being notoriously difficult to study directly, investigation of related classes of quadratic algebras can provide insight leading to a greater understanding of division algebras. The reversible and von-Neumann finite quadratic algebras are two such classes. While both properly contain the class of quadratic division algebras, they are also sufficiently narrowly defined to make discernible certain particular structural properties of these classes of algebras.

Let $F$ be a field. An $F$-algebra is a vector space $A$ over $F$ endowed with a bilinear multiplication map $A \times A \rightarrow A,(a, b) \mapsto a b$. An $F$-algebra $A$ is said to be unital if it possesses a multiplicative identity element $1=1_{A}$. In this article, we will consider the problem of characterising von-Neumann finiteness and reversibility for the classes of nonassociative algebras defined below.

Definition 1.1 An $F$-algebra $A$ is said to be

(a) alternative if it satisfies the identities $a^{2} b=a(a b)$ and $a b^{2}=(a b) b$ for all $a, b \in A$. Equivalently, every subalgebra of $A$ generated by at most two elements is associative [21, Theorem 3.1].

(b) flexible if it satisfies the identity $a(b a)=(a b) a$ for all $a, b \in A$.

(c) quadratic if it is unital and the elements $1, a, a^{2}$ are linearly dependent for all $a \in A$.

(d) involutive if it is unital and there exists an anti-automorphism $\sigma$ of $A$ such that $\sigma^{2}=$ $\mathbb{I}_{A}, a+\sigma(a) \in F 1$ and $a \sigma(a) \in F 1$ for all $a \in A$. We will use the notation $\sigma(a)=\bar{a}$ for the involution in $A$. For any $a \in A$, the scalars $\operatorname{tr}(a)=a+\bar{a}$ and $\mathrm{n}(a)=a \bar{a}$ are called the trace and the norm of $A$, respectively.

From here on, all algebras will be assumed to be unital (but not necessarily associative). Throughout, $F$ denotes a field, and $A$ an $F$-algebra.

To every quadratic form $q: V \rightarrow F$ on a vector space $V$ over $F$ is associated a symmetric bilinear form $\langle x, y\rangle=\langle x, y\rangle_{q}=q(x+y)-q(x)-q(y)$. The radical of $q$ is the subspace $V^{\perp}=\left\{x \in V \mid\langle x, V\rangle_{q}=0\right\}$ of $V$. The form $q$ is said to be non-degenerate if either $V^{\perp}=0$ or $\operatorname{dim}\left(V^{\perp}\right)=1$ and $q\left(V^{\perp}\right) \neq 0$ (the latter case occurs only for char $F=2$ ). A non-zero element $v \in V$ is called isotropic if $q(v)=0$ and anisotropic otherwise. The form $q$ is said to be isotropic, respectively anisotropic, if $V$ contains, respectively does not 
contain, an isotropic element. A subspace $U$ of $V$ such that $q(U)=0$ is said to be totally isotropic.

A Hurwitz algebra is an algebra $A$ possessing a non-degenerate quadratic form $\mathrm{n}: A \rightarrow$ $F$ satisfying $\mathrm{n}(a b)=\mathrm{n}(a) \mathrm{n}(b)$ for all $a, b \in A$. The form $\mathrm{n}$ is uniquely determined by the algebra structure of $A$, and every non-zero algebra morphism between Hurwitz algebras is orthogonal. A Hurwitz algebra $A$ has zero-divisors if and only if its quadratic form $\mathrm{n}$ is isotropic. If this is the case, $A$ is said to be split. Over any ground field, there are precisely three isomorphism classes of split Hurwitz algebra, one in each dimension 2, 4 and 8, consecutively embedded into each other. The 4-dimensional split Hurwitz $F$-algebra is the $2 \times 2$ matrix algebra over $F$. A Hurwitz algebra is commutative if and only if its dimension is at most 2, and associative if and only if its dimension is at most 4. All Hurwitz algebras are alternative. For further details, we refer to [11, Chapter VIII].

We denote by $H=F^{2 \times 2}$ the 4-dimensional split Hurwitz algebra over $F$, and by $U \subset H$ the 3-dimensional subalgebra of upper triangular matrices.

Given an involutive algebra $B$ and a non-zero scalar $\mu$, a new algebra $\mathcal{C D}_{\mu}(B)$ is constructed as follows: as a vector space, $\mathcal{C D}_{\mu}(B)=B \times B$, with multiplication defined by

$$
(a, b)(c, d)=(a c+\mu \bar{d} b, d a+b \bar{c})
$$

for all $a, b, c, d \in B$. The algebra $\mathcal{C D}_{\mu}(B)$ is said to be obtained from $B$ by the CayleyDickson doubling process. It is again involutive, with involution given by $\overline{(a, b)}=(\bar{a},-b)$, and flexible if and only if $B$ is flexible [17, Sec. 6]. Commonly, $B$ is identified with the subalgebra $B \times 0$ of $\mathcal{C D}_{\mu}(B)$, and setting $\ell=(0,1) \in B \times B$ gives an orthogonal decomposition of vector spaces $\mathcal{C D}_{\mu}(B)=B \oplus B \ell$.

Starting from the algebra $F$ with trivial involution, one obtains a class of flexible involutive algebras of dimensions $2^{m}, m \geqslant 1$, by successive application of the Cayley-Dickson construction. In this paper, we shall refer to the algebras constructed in this way as CayleyDickson algebras. When char $F \neq 2$, the Cayley-Dickson algebras of dimension at most eight are precisely the Hurwitz algebras over $F$ [1]. (Hurwitz algebras over fields of characteristic 2 can also be constructed by the Cayley-Dickson process by starting from a so-called quadratic étale algebra, but this construction will play no role in the present paper.)

The present article is organised as follows. In Section 2, we summarise our main results. Section 3 contains some general background information and lemmata, and Section 4 the proofs of the main results. Finally, in Section 5 we give some examples illuminating different aspects of the theory.

A note on conventions: we use words as non-commutative and non-associative in the strict sense: a non-commutative algebra is one that does not satisfy the commutative law, etc.

\section{Summary of Our Results}

Our first theorem, concerning alternative algebras, is rather straightforward from existing theory.

Theorem 2.1 (Propositions 4.1-4.2) (a) Every finite-dimensional alternative algbra is von-Neumann finite.

(b) Every reversible alternative algebra is von-Neumann finite. 
(c) A Hurwitz algebra A is reversible if and only if either its quadratic form is anisotropic, or $\operatorname{dim} A \leqslant 2$.

Note that, by Theorem 2.1(a), all Hurwitz algebras are von-Neumann finite.

The next theorem summarises properties of algebras that are both flexible and quadratic. Such algebras are in particular involutive, by Lemma 3.4(a) in the next section, and hence equipped with a norm.

Theorem 2.2 (Propositions 4.3,4.4 and 4.13) (a) Every algebra without zero divisors, that is either flexible or quadratic, is von-Neumann finite.

(b) Assume that char $F \neq 2$, and let $A$ be flexible and quadratic. If the norm of $A$ is nondegenerate on every 3-dimensional subalgebra of $A$, then $A$ is von-Neumann-finite and reversible.

(c) Assume that $F$ is algebraically closed, char $F \neq 2$, and that $A$ is flexible and quadratic. Then $A$ is reversible if and only if either of the following conditions holds:

(i) A is commutative;

(ii) $A=F 1 \oplus V$, where $V$ is an anti-commutative ideal in $A$, and the linear map $\mathrm{L}_{u}: V \rightarrow V, v \mapsto u v$ is nilpotent for all $u \in V$.

In relation to Theorem 2.2(a), note that every algebra without zero divisors is reversible. We also point out that Theorem 2.2(b) in particular applies to every flexible quadratic $F$ algebra with anisotropic norm. This immediately implies the first first part of our next theorem.

Theorem 2.3 (Corollary 4.5, Corollary 4.7) Assume that char $F \neq 2$.

(a) All Cayley-Dickson algebras with anisotropic norm are von-Neumann finite and reversible.

(b) A Cayley-Dickson algebra with isotropic norm is reversible if and only if its dimension is at most two.

It is not clear to the authors if there exist Cayley-Dickson algebras with isotropic norm that are not von-Neumann finite. By Theorem 2.4(a) below, such an algebra would need to have a 3-dimensional subalgebra that is neither commutative nor associative. We also remark that when char $F \neq 2$, Theorem 2.1(c) is a consequence of Theorem 2.3(b).

Theorem 2.4 (Propositions 4.10 and 4.11) Let $A$ be an involutive algebra, and assume that $\operatorname{char} F \neq 2$.

(a) The algebra A is von-Neumann finite if and only if every 3-dimensional subalgebra of $A$ is either commutative or associative.

(b) The algebra $A$ is reversible if and only if every 3-dimensional subalgebra of $A$ is commutative.

Actually, every non-commutative associative involutive algebra of dimension three over $F$ is isomorphic to the algebra $U$. 


\section{Preliminaries}

First, observe that the classes of von-Neumann finite respectively reversible algebras are closed under taking subalgebras. Since matrix algebras are von-Neumann finite, and every finite-dimensional associative algebra can be embedded in a matrix algebra, we have the following (well known) result.

Lemma 3.1 Every finite-dimensional associative F-algebra is von-Neumann finite.

Lemma 3.2 The following statements are equivalent.

(i) The algebra A is von-Neumann finite (respectively, reversible);

(ii) every subalgebra of A generated by at most two elements is von-Neumann finite (reversible);

(iii) any two elements $a, b \in A$ satisfying $a b \in F \backslash\{0\}(a b=0)$ commute with each other.

Proof If $A$ is von-Neumann finite then so is every subalgebra of $A$. Hence (i) $\Rightarrow$ (ii). Assuming (i), and taking $a, b \in A$ such that $a b=\mu \in F \backslash\{0\}$, we have $\left(\mu^{-1} a\right) b=1$. By assumption, the subalgebra generated by $\mu^{-1} a$ and $b$ is von-Neumann finite, and hence $b\left(\mu^{-1} a\right)=1=\left(\mu^{-1} a\right) b$, implying that $a b=b a$. This shows that (ii) $\Rightarrow($ iii). Finally, if (iii) holds and $a b=1$, then $b a=a b=1$, so $A$ is von-Neumann finite.

The reversible case is analogous.

We now turn our attention to involutive and quadratic algebras.

Lemma 3.3 Let $A$ be a quadratic algebra.

(a) Let $a, b \in A$ and $B=\operatorname{span}\{1, a, b\}$. If $a b \in B$ then $B$ is a subalgebra of $A$.

(b) The algebra $A$ is von-Neumann finite (respectively, reversible) if and only if every 3-dimensional subalgebra of $A$ is von-Neumann finite (reversible).

(c) The algebra $A$ is von-Neumann finite and reversible if and only if every 3-dimensional subalgebra of $A$ is commutative.

Proof (a) Since $A$ is quadratic, $a^{2} \in \operatorname{span}\{1, a\} \subset B$, and similarly $b^{2},(a+b)^{2} \in B$. Moreover, $b a=(a+b)^{2}-a^{2}-b^{2}-a b \in B$, so $B \subset A$ is a subalgebra.

(b) The "only if" part is immediate. For the converse, assume that every 3-dimensional subalgebra of $A$ is von-Neumann finite, and let $a, b \in A$ such that $a b=1$. By (a), $B=\operatorname{span}\{1, a, b\}$ is a subalgebra of $A$ of dimension at most 3 . If $\operatorname{dim} B<3$ then $B$ is commutative and thus von-Neumann finite, and if $\operatorname{dim} B=3$ then $B$ is von-Neumann finite by assumption. Hence, $b a=1$, and so $A$ is von-Neumann finite. Reversibility is proved analogously.

(c) In view of (b), we need to show that a 3-dimensional quadratic algebra is vonNeumann finite and reversible if and only if it is commutative. Clearly, any commutative algebra is both von-Neumann finite and reversible. Let $B=\operatorname{span}\{1, a, b\}$ be a von-Neumann-finite reversible quadratic algebra, and $a b=\alpha+\beta a+\gamma b$, for some $\alpha, \beta, \gamma \in F$. Then

$$
(a-\gamma)(b-\beta)=a b-\beta a-\gamma b+\beta \gamma=\alpha+\beta \gamma \in F,
$$


implying that $(a-\gamma)(b-\beta)=(b-\beta)(a-\gamma)$ since $B$ is von-Neumann finite and reversible. Now $0=(a-\gamma)(b-\beta)-(b-\beta)(a-\gamma)=a b-b a$, so $a b=b a$ and $B=\operatorname{span}\{1, a, b\}$ is commutative.

Note that in an involutive algebra, the identity $\mathrm{n}(a)=a \bar{a}=\bar{a} a$ holds: $\operatorname{as} \operatorname{tr}(a) \in F$ it follows that $\operatorname{tr}(a) a=a \operatorname{tr}(a)$, and thus

$$
a \bar{a}=a(\operatorname{tr}(a)-a)=\operatorname{tr}(a) a-a^{2}=(\operatorname{tr}(a)-a) a=\bar{a} a .
$$

Equation 1 also implies that $a^{2}-\operatorname{tr}(a) a+\mathrm{n}(a)=0$ so, in particular, every involutive algebra is quadratic.

In the paper [19], Osborn developed the fundamentals of a theory for quadratic algebras over fields of characteristic different from 2. For such an algebra $A$, set

$$
\operatorname{Im} A=\left\{u \in A \backslash F 1 \mid u^{2} \in F 1\right\} \cup\{0\} .
$$

Then $A$ decomposes as a vector space as $A=F \oplus \operatorname{Im} A=F 1 \oplus \operatorname{Im} A$. This decomposition defines an $F$-bilinear form $(\cdot, \cdot)$ and an anti-commutative multiplication $\times$ on $\operatorname{Im} A$, by the formula $u v=(u, v)+u \times v \in F \oplus \operatorname{Im} A$ for all $u, v \in \operatorname{Im} A$. Multiplication in $A=F \oplus \operatorname{Im} A$ can now be written as

$$
(\alpha, u)(\beta, v)=(\alpha \beta+(u, v), \alpha v+\beta u+u \times v),
$$

for $\alpha, \beta \in F, u, v \in \operatorname{Im} A$. A linear map $\sigma: A \rightarrow A, a \mapsto \bar{a}$ is defined by $\sigma(\alpha, u)=$ $(\alpha,-u)$ and, similarly to the involutive case, we have $\operatorname{tr}(a)=a+\bar{a}=2 \alpha \in F, \mathrm{n}(a)=$ $a \bar{a}=\bar{a} a=\alpha^{2}-(u, u) \in F$ and $a^{2}-\operatorname{tr}(a) a+\mathrm{n}(a)=0$ for $a=(\alpha, u) \in A$. Moreover, $(u, v)=\frac{1}{2} \operatorname{tr}(u v)$ for all $u, v \in \operatorname{Im}(A)$.

Lemma 3.4 (Osborn [19, p 203]) Let A be a quadratic algebra, and char $F \neq 2$.

(a) The algebra $A$ is involutive if and only if $(\cdot, \cdot)$ is symmetric.

(b) The algebra $A$ is flexible if and only if the bilinear form $(\cdot, \cdot)$ is symmetric and $(u, u \times$ $v)=0$ holds for all $u, v \in \operatorname{Im} A$.

In particular, every flexible quadratic $F$-algebra is involutive.

For $A$ quadratic and char $F \neq 2$, we denote by $\mathrm{L}_{u}^{\times}$the linear map $\operatorname{Im} A \rightarrow \operatorname{Im} A, v \mapsto$ $u \times v$.

Lemma 3.5 Let $A$ be a quadratic $F$-algebra, and char $F \neq 2$.

(a) Every 3-dimensional subalgebra of $A$ is commutative if and only if the condition $u \times$ $v \in \operatorname{span}\{u, v\}$ implies $(u, v)=(v, u)$ and $u \times v=0$ for all $u, v \in \operatorname{Im} A$.

(b) Assume additionally that $F$ is algebraically closed, and A involutive. Then every 3dimensional subalgebra of $A$ is commutative if and only if the $m a p \mathrm{~L}_{u}^{\times}$is nilpotent for all $u, v \in \operatorname{Im} A$.

Proof (a) For any $u, v \in \operatorname{Im} A$, the subspace $B=\operatorname{span}\{1, u, v\} \subset A$ is a subalgebra if and only if $u \times v \in \operatorname{span}\{u, v\}$. On the other hand, $u v-v u=((u, v)-(v, u), 2 u \times v) \in$ $F \oplus \operatorname{Im} A$. Consequently, $B$ is commutative if and only if $(u, v)=(v, u)$ and $u \times v=0$.

(b) Since $A$ is involutive, the bilinear form $(\cdot, \cdot)$ is symmetric by Lemma 3.4(a). Moreover, as the product $\times$ on $\operatorname{Im} A$ is anti-commutative, the condition $u, v \in \operatorname{span}\{u, v\} \Rightarrow$ 
$u \times v=0$ for all $u, v \in \operatorname{Im} A$ is equivalent to that, for all $u \in \operatorname{Im} A$, the map $\mathrm{L}_{u}^{\times}$: $\operatorname{Im} A \rightarrow \operatorname{Im} A$ has no non-zero eigenvalues. By the Jordan normal form theorem, this is equivalent to $\mathrm{L}_{u}^{\times}$being nilpotent for all $u \in \operatorname{Im} A$. Hence, the result follows from Lemma 3.5(a).

For the case char $F \neq 2$, we shall need the following fact about Cayley-Dickson algebras, which is a consequence of the fact that their norms are Pfister forms [12, Chapter X].

Lemma 3.6 ([12, Theorem X.1.7]) Assume that char $F \neq 2$ and let A be a Cayley-Dickson algebra of even dimension $2 m$, with isotropic norm. Then A has a totally isotropic subspace of dimension $m$.

\section{Proofs of Our Results}

\subsection{Proof of Theorem 2.1}

Proposition 4.1 Every alternative algebra that is either finite dimensional or reversible is von-Neumann finite.

Proof Let $A$ be an alternative algebra, and $B \subset A$ a subalgebra generated by at most two elements. If $A$ is finite dimensional or reversible then so is $B$. If $B$ is finite dimensional then it is von-Neumann finite by Lemma 3.1 ; if it is reversible then the same conclusion follows from the argument given in the introduction. Thus, by Lemma 3.2, the algebra $A$ is von-Neumann finite.

Proposition 4.2 Every Hurwitz algebra with anisotropic norm is reversible. A split Hurwitz algebra is reversible if and only if its dimension is less than or equal to 2.

Proof Hurwitz algebras with anisotropic norm have no zero divisors, and Hurwitz algebras of dimension at most 2 are commutative. Hence, all algebras of either type are reversible. If $A$ is a split Hurwitz algebra of dimension at least 4, then it contains a split quaternion subalgebra, that is, an algebra isomorphic to $H$. Since $H$ is not reversible, neither is $A$ in this case.

\subsection{Proof of Theorem 2.2(a,b)}

Proposition 4.3 Every algebra without zero divisors, that is either flexible or quadratic, is von-Neumann finite.

Proof Let $A$ be an algebra without zero divisors. If $A$ is flexible and $a b=1$ then $a=$ $(a b) a=a(b a)$, whence $a(1-b a)=0$ and $b a=1$. If $A$ is quadratic then $\operatorname{span}\{1, a\}$ is a field for every $a \in A$, and hence there exists an element $\tilde{a} \in \operatorname{span}\{1, a\}$ such that $a \tilde{a}=\tilde{a} a=1$. Now, if $a b=1$ then $a(b-\tilde{a})=a b-a \tilde{a}=1-1=0$, implying that $b=\tilde{a}$ and hence $b a=1$.

For the remainder of Section 4 , we assume that char $F \neq 2$. 
Proposition 4.4 Let $A$ be a flexible quadratic $F$-algebra. If the norm $\mathrm{n}$ of $A$ is nondegenerate on every 3-dimensional subalgebra of $A$, then $A$ is von-Neumann finite and reversible.

Proof Any 3-dimensional subalgebra $B \subset A$ can be written as $B=\operatorname{span}\{1, u, v\}$, where $u, v \in \operatorname{Im} A$ are anisotropic vectors. Since $A$ is flexible, the bilinear form $(\cdot, \cdot)$ on $\operatorname{Im} A$ is symmetric by Lemma 3.1(b), moreover, $\mathrm{n}(w)=-(w, w)$ and $\langle w, z\rangle_{\mathrm{n}}=-2(w, z)$ for all $w, z \in \operatorname{Im} A$. Again by Lemma 3.4(b), we have $(u, u \times v)=(v, u \times v)=0$ implying that $\langle u, u \times v\rangle=\langle v, u \times v\rangle=0$ and, consequently, $u \times v \in \operatorname{Im} B \cap(\operatorname{Im} B)^{\perp}=0$. Thus $B$ is commutative, by Lemma 3.5(a). Lemma 3.3(c) gives the result.

\subsection{Proof of Theorem 2.3}

Since Cayley-Dickson algebras are flexible and quadratic, Proposition 4.4 implies the following result.

Corollary 4.5 All Cayley-Dickson algebras with anisotropic norm are von-Neumann finite and reversible.

Theorem 2.3(b) is a consequence of the following proposition.

Proposition 4.6 Let A be a Cayley-Dickson algebra of dimension at least 4 with isotropic norm. Then A has a subalgebra isomorphic to the split quaternion algebra $\mathrm{H}$.

Proof By construction, the algebra $A$ contains a subalgebra of the form $B^{\prime}=\mathcal{C D}_{\mu}(B)=$ $B \oplus B \ell$, where $B$ is a Cayley-Dickson algebra with anisotropic norm, $\mu \in F \backslash\{0\}$, and the norm of $B^{\prime}$ is isotropic. Set $m=\operatorname{dim} B$. If $m=1$ then $B^{\prime} \simeq F \times F$ and, since $\operatorname{dim} A \geqslant 4$, there exists a subalgebra of $A$ of the form $\mathcal{C D}_{v}\left(B^{\prime}\right) \simeq \mathcal{C D}_{v}(F \times F) \simeq H, v \in F \backslash 0$.

Assume instead that $m \geqslant 2$. By Lemma 3.6, the $(2 m)$-dimensional quadratic space $B^{\prime}$ contains a totally isotropic subspace $U$ of dimension $m$. It follows that the $(m+1)$ dimensional subspace $B \oplus F \ell$ of $B^{\prime}$ intersects $U$ non-trivially, and hence $B \oplus F \ell$ contains an isotropic vector $v$. Let $b \in B$ be such that $v-b \in F \ell$, and let $C \subset B$ be a 2-dimensional subalgebra containing $b$. Then $C^{\prime}=C \oplus C \ell \subset B^{\prime}$ is a subalgebra of dimension 4 , and $C^{\prime} \simeq \mathcal{C} \mathcal{D}_{\mu}(C)$. Hence $C^{\prime}$ is a quaternion subalgebra of $A$, and since $v \in C^{\prime}$ is an isotropic element, it follows that $C^{\prime} \simeq H$.

Corollary 4.7 A Cayley-Dickson algebra with isotropic norm is reversible if and only if its dimension is at most two.

\subsection{Proof of Theorem 2.2(c) and Theorem 2.4}

We start by proving Theorem 2.4.

Lemma 4.8 Let $A$ be an involutive algebra.

(a) The identity $\overline{a b}-b a=\operatorname{tr}(a) \operatorname{tr}(b)-\operatorname{tr}(a) b-\operatorname{tr}(b) a$ holds for all $a, b \in A$.

(b) The algebra $A$ is von-Neumann finite if and only if the condition ab $\in \backslash\{0\}$ implies that either $a, b \in \operatorname{Im} A$ or $1, a, b$ are linearly dependent. 
(c) The algebra $A$ is reversible if and only if $a b=0$ implies that either $a, b \in \operatorname{Im} A$ or $1, a, b$ are linearly dependent.

Proof (a) This is a straightforward calculation:

$$
\overline{a b}-b a=\bar{b} \bar{a}-b a=(\operatorname{tr}(b)-b)(\operatorname{tr}(a)-a)-b a=\operatorname{tr}(b) \operatorname{tr}(a)-\operatorname{tr}(b) a-\operatorname{tr}(a) b .
$$

(b,c) We shall prove that if $a b \in F$, then $a b=b a$ if and only if either 1, $a, b$ are linearly dependent or $a, b \in \operatorname{Im} A$. This, together with Lemma 3.2, implies the result.

Let $a b \in F$. Then $\overline{a b}=a b$, so $a b-b a=\overline{a b}-b a=\operatorname{tr}(a) \operatorname{tr}(b)-\operatorname{tr}(a) b-\operatorname{tr}(b) a$ by (a). Hence, if $a b=b a$ then either $1, a, b$ are linearly dependent, or $\operatorname{tr}(a)=\operatorname{tr}(b)=0$, that is $a, b \in \operatorname{Im} A$. Conversely, if $\operatorname{tr}(a)=\operatorname{tr}(b)=0$ then clearly $a b-b a=0$. If instead $1, a, b$ are linearly dependent, then these elements are contained in a subalgebra of dimension at most two, and hence commute with each other.

Lemma 4.9 Let $B$ be a 3-dimensional non-commutative involutive algebra. Then there exists a basis $u, v$ of $\operatorname{Im} B$ such that $u \times v=u$ and either $(u, u)=0$ or $(u, v)=0$.

Proof Since $B$ is involutive, the bilinear form $(\cdot, \cdot)$ is symmetric. Consequently, commutativity of $B$ is equivalent to the condition that $x \times y=0$ for all $x, y \in \operatorname{Im} B$. As $B$ is not commutative, the product $\times$ on $\operatorname{Im} B$ is non-trivial, so there exist $x, y \in \operatorname{Im} B$ such that $x \times y \neq 0$. Set $u=x \times y$. Using the anti-commutaticity of $\times$ we compute

$(\alpha x+\beta y) \times(\gamma x+\delta y)=\alpha \gamma x \times x+\alpha \delta x \times y+\beta \gamma y \times x+\beta \delta y \times y=(\alpha \delta-\beta \gamma) x \times y=(\alpha \delta-\beta \gamma) u$.

This means that $(\operatorname{Im} B) \times(\operatorname{Im} B)=\operatorname{span}\{u\}$, and that $w \times z=0$ if and only if $w, z \in \operatorname{Im} A$ are linearly dependent. Now, if $(u, u) \neq 0$ then there exists a vector $v \in \operatorname{Im} B$ such that $(u, v)=0$ and $u \times v=u$, and thus $u, v$ is a basis of $\operatorname{Im} B$ with the required properties. In case $(u, u)=0$, any $v \in \operatorname{Im} B$ such that $u \times v=u$ will do.

Proposition 4.10 Let $A$ be an involutive algebra. The following statements are equivalent.

(i) The algebra $A$ is von-Neumann finite;

(ii) every 3-dimensional subalgebra of $A$ is either commutative or associative;

(iii) every 3-dimensional subalgebra of $A$ is either commutative or isomorphic to the algebra $U$ of upper triangular $2 \times 2$ matrices.

Proof As all commutative algebras and all finite-dimensional associative algebras are vonNeumann finite, the implication (ii) $\Rightarrow$ (i) is immediate from Lemma 3.3(b). Moreover, we have (iii) $\Rightarrow$ (ii) since the algebra $U$ is associative. To prove (i) $\Rightarrow$ (iii), by Lemma 3.3(b), it suffices to show that if $B$ is a 3-dimensional von-Neumann-finite non-commutative involutive algebra, then $B \simeq U$.

By Lemma 4.9, there exists a basis $u, v$ of $\operatorname{Im} B$ such that $u \times v=u$. For this basis, we have

$$
(0, u)(-1, v)=((u, v),-u+u \times v)=(u, v) \in F .
$$


Moreover, the elements $1,(0, u),(-1, v)$ of $B$ are linearly independent, $(-1, v) \notin \operatorname{Im} B$ and $B$ is von-Neumann finite. Thus Lemma 4.8(b) implies that $(u, v)=(0, u)(-1, v)=0$. Similarly, again applying Lemma 4.8(b),

$$
\begin{aligned}
(1, u+v)(-1, v) & =(-1+(v, v), v-u-v+u \times v)=(v, v)-1 \in F \Rightarrow & & (v, v)=1, \text { and } \\
(1, u+v)(0, u) & =((u, u), u+v \times u)=(u, u) \in F & \Rightarrow & (u, u)=0 .
\end{aligned}
$$

From the identities $u \times v=u,(u, u)=(u, v)=0,(v, v)=1$, and Eq. 2, it is easy to work out the multiplication table of $B: u^{2}=0, v^{2}=1$ and $u v=u=-v u$. This coincides with the multiplication table of $U$ given in Table 2 (see Example 5.1 below), consequently, $B \simeq U$.

Proposition 4.11 Let $A$ be an involutive algebra. The following statements are equivalent.

(i) The algebra $A$ is reversible.

(ii) Every 3-dimensional subalgebra of $A$ is commutative.

Proof Again, the implication (ii) $\Rightarrow$ (i) follows directly from Lemma 3.3(b). For the converse, assume that there exists a reversible involutive algebra $B$ of dimension 3 that is not commutative. Then, by Lemma 4.9, there exist $u, v \in \operatorname{Im} B$ such that $u \times v=u$ and either $(u, u)=0$ or $(u, v)=0$. Computing

$$
(1, v)(0, u)=((u, v), u+v \times u)=(u, v) \in F
$$

and invoking Lemma 4.8(c), we get that $(u, v) \neq 0$ and, consequently, $(u, u)=0$. Now, similarly,

$$
\begin{aligned}
(1, u+v)(1, u-v) & =(1-(v, v),(u-v)+(u+v)+(u+v) \times(u-v)) \\
& =(1-(v, v), 2 u-u \times v+v \times u)=1-(v, v) \in F
\end{aligned}
$$

and thus $1-(v, v) \neq 0$ by Lemma 4.8(c). Now, setting $y=(1-(v, v)) u+(u, v) v$ we get

$$
\begin{aligned}
& (v, y)=(1-(v, v))(u, v)+(u, v)(v, v)=(u, v), \\
& v \times y=(1-(v, v)) v \times u=((v, v)-1) u,
\end{aligned}
$$

and hence

$$
\begin{aligned}
(1, v)(-(u, v), y) & =(-(u, v)+(v, y), y-(u, v) v+v \times y) \\
& =(0,(1-(v, v)) u+(u, v) v-(u, v) v+((v, v)-1) u)=0 .
\end{aligned}
$$

Note that since $(1-(v, v)) \neq 0$, the elements $v$ and $y$ are linearly independent. As $(1, v) \notin \operatorname{Im} B$, Lemma 4.8 (c) implies that $B$ cannot be reversible, a contradiction. Hence $B$ is commutative.

As the norm of $U$ is isotropic, Proposition 4.1. and Proposition 4.11 together have the following consequences.

Corollary 4.12 Let $A$ be an involutive algebra.

(a) If $A$ is reversible then it is von-Neumann finite.

(b) If $A$ is von-Neumann finite and the norm of $A$ is anisotropic, then $A$ is reversible.

The following result establishes the proof of Theorem 2.2(c). 
Proposition 4.13 Assume that $F$ is algebraically closed, and let $A$ be a flexible and quadratic F-algebra. Then $A$ is reversible if and only if one of the following conditions holds:

(i) $u \times v=0$ for all $u, v \in \operatorname{Im} A$, that is, $A$ is commutative;

(i) $\mathrm{n}(u)=0$ for all $u \in \operatorname{Im} A$, and the map $\mathrm{L}_{u}^{\times}: \operatorname{Im} A \rightarrow \operatorname{Im} A, v \mapsto u \times v$ is nilpotent for all $u \in \operatorname{Im} A$.

Note that in the latter case, the subspace $\operatorname{Im} A$ is an ideal in $A$, and $A$ is obtained from $\operatorname{Im} A$ by adjoining an identity element. Hence, reversible algebras of this type are given by anti-commutative (non-unital) algebras $V$ satisfying the nilpotency condition specificed in (ii). This holds, for example, if $V$ is a nilpotent Lie algebra.

Proof Combining Proposition 4.11 with Lemma 3.5(b), we see that $A$ is reversible if and only if $\mathrm{L}_{u}^{\times}$is nilpotent for all $u \in \operatorname{Im} A$. Clearly, this holds if $A$ satisfies either of the conditions (i) and (ii). For the converse implication, assume that $\mathrm{L}_{u}^{\times}$is nilpotent for all $u \in$ $\operatorname{Im} A$. We shall show that for any anisotropic $w \in \operatorname{Im} A$, the identity $\mathrm{L}_{w}^{\times}=0$ holds. Then, since the anisotropic vectors form a Zariski-open subset of $\operatorname{Im} A$, whereas the condition $\mathrm{L}_{w}^{\times}=0$ is closed, it follows that either $\mathrm{n}=0$ or $\mathrm{L}_{w}=0$ for all $w \in \operatorname{Im} A$.

First, linearising the identity $(u, u \times v)=0$ in Lemma 3.4(b) gives the equivalent condition

$$
(u \times v, w)=(u, v \times w)
$$

for all $u, v, w \in \operatorname{Im} A$. Assume that $w \in \operatorname{Im} A$ is anisotropic, $0 \leqslant m<\operatorname{dim}(\operatorname{Im} A)$, and that there exist linearly independent anisotropic vectors $w=v_{1}, v_{2}, \ldots, v_{m}$, such that $w \times v_{i}=0$ for all $i \leqslant m$. Set $W_{m}=\operatorname{span}\left\{v_{1}, \ldots, v_{m}\right\}^{\perp} \subset \operatorname{Im} A$. Then, for all $u \in \operatorname{Im} A$,

$$
0=\left(w \times v_{i}, u\right)=-\left(v_{i} \times w, u\right)=-\left(v_{i}, w \times u\right),
$$

that is, $\mathrm{L}_{w}^{\times}(\operatorname{Im} A) \subset W_{m}$. In particular, the subspace $W_{m}$ is invariant under $\mathrm{L}_{w}^{\times}$. The map $\mathrm{L}_{w}^{\times}$ being nilpotent, it means that there exists a non-zero element $z \in W_{m}$ such that $\mathrm{L}_{w}^{\times}(z)=0$.

Now, set $v_{m+1}=z$ if $\mathrm{n}(z) \neq 0$, and $v_{m+1}=z+w$ if $\mathrm{n}(z)=0$. In either case, the elements $v_{1}, \ldots, v_{m+1} \in \operatorname{Im} A$ are linearly independent, anisotropic, and contained in $\operatorname{ker}\left(\mathrm{L}_{w}^{\times}\right)$. By induction, $\operatorname{ker}\left(\mathrm{L}_{w}^{\times}\right)=\operatorname{Im} A$.

\section{Examples}

We start by mentioning two particular, associative algebras that are of certain importance for our study.

Example 5.1 Let char $F \neq 2$. The split quaternion $F$-algebra $H$ is, as already mentioned, isomorphic to the algebra $F^{2 \times 2}$ of $2 \times 2$ matrices with entries in $F$. Choosing

$$
i=\left(\begin{array}{cc}
-1 & 0 \\
0 & 1
\end{array}\right), \quad j=\left(\begin{array}{ll}
0 & 1 \\
1 & 0
\end{array}\right), \quad \text { and } \quad k=\left(\begin{array}{cc}
0 & 1 \\
-1 & 0
\end{array}\right)
$$

gives a basis $\underline{e}=(1, i, j, k)$ of $H$, with multiplication as in Table 1 .

It is easy to see that $\operatorname{Im} H=\operatorname{span}\{i, j, k\}$, and that $\underline{e}$ is an orthogonal basis of $H$. Indeed, since $\langle x, y\rangle=\mathrm{n}(x+y)-\mathrm{n}(x)-\mathrm{n}(y)=x y+y x$ for all $x, y \in \operatorname{Im} H$, a subset of $\operatorname{Im} H$ is orthogonal if and only its elements pair-wise anti-commute - as do $i, j, k$. 
Table 1 Multiplication in $H$

\begin{tabular}{lllll}
\hline & 1 & $\mathrm{i}$ & $\mathrm{j}$ & $\mathrm{k}$ \\
\hline 1 & 1 & $\mathrm{i}$ & $\mathrm{j}$ & $\mathrm{k}$ \\
$\mathrm{i}$ & $\mathrm{i}$ & 1 & $\mathrm{k}$ & $\mathrm{j}$ \\
$\mathrm{j}$ & $\mathrm{j}$ & $-\mathrm{k}$ & 1 & $-\mathrm{i}$ \\
$\mathrm{k}$ & $\mathrm{k}$ & $-\mathrm{j}$ & $\mathrm{i}$ & -1 \\
\hline
\end{tabular}

Now consider the subalgebra $U$ of $F^{2 \times 2}$, consisting of all upper triangular matrices. Being a subalgebra of an involutive associative algebra, $U$ is again involutive and associative. We choose a basis $\underline{f}=(1, u, v)$, where

$$
u=\frac{1}{2}(j+k)=\left(\begin{array}{ll}
0 & 1 \\
0 & 0
\end{array}\right) \quad \text { and } \quad v=i=\left(\begin{array}{cc}
-1 & 0 \\
0 & 1
\end{array}\right) .
$$

Again, $\underline{f}$ is orthogonal, and $\operatorname{Im} U=\operatorname{span}\{u, v\}$. The multiplication of elements in $\underline{f}$ is given by Table 2 .

As $(1+v) u=0$ but $u(1+v)=2 u \neq 0$, the algebras $U$ and $H$ are not reversible. However, being finite-dimensional associative algebras, they are von-Neumann finite.

The following examples demonstrate the necessity of some of the assumptions in our main theorems. First we give an example of a flexible quadratic algebra with non-degenerate norm that is neither reversible nor von-Neumann finite. The existence of such an algebra implies that the hypothesis in Theorem 2.2(b), that the norm is non-degenerate on every 3-dimensional subalgebra, cannot be replaced by the weaker condition of the norm being non-degenerate on $A$ itself.

Example 5.2 Assume that char $F \neq 2$, and let $H$ be the split quaternion algebra with basis $\underline{e}=(1, i, j, k)$ as in Example 5.1.

Set $A=H \oplus F l$, where $l(\operatorname{Im} H)=(\operatorname{Im} H) l=0$ and $l^{2}=-1$. This defines $A$ as a quadratic algebra with $\operatorname{Im} A=\operatorname{span}\{i, j, k, l\}$. Since $H$ is associative and thus in particular flexible, the bilinear form $(\cdot, \cdot)_{H}$ on $H$ is symmetric, and $(u, u \times v)_{H}=0$ for all $u, v \in$ $\operatorname{Im} H$. By the construction, it follows that also $(\cdot, \cdot)_{A}$ is symmetric, and $(w, w \times z)_{A}=0$ for all $w, z \in \operatorname{Im} A$. Hence, Lemma 3.4 implies that $A$ is flexible and thus, in particular, involutive. The elements $1, i, j, k, l$ constitute an orthogonal basis of $A$, so the norm on $A$ is non-degenerate.

Set $x=i+l, y=j+k$, and $B=\operatorname{span}\{1, x, y\} \subset A$. We have $x y=(i+l)(j+k)=i j+i k=k+j=y \quad$ and $\quad y x=(j+k)(i+l)=j i+k i=-k-j=-y$ so $B$ is a 3-dimensional non-commutative subalgebra of $A$. Moreover, $x^{2}=i^{2}+l^{2}=$ $1-1=0$, so $x^{2} y=0$, whereas $x(x y)=x y=y \neq x^{2} y$. Hence $B$ is not associative either.

Table 2 Multiplication in $U$

\begin{tabular}{lllr}
\hline$\cdot$ & 1 & $\mathrm{u}$ & $\mathrm{v}$ \\
\hline 1 & 1 & $\mathrm{u}$ & $\mathrm{v}$ \\
$\mathrm{u}$ & $\mathrm{u}$ & 0 & $\mathrm{u}$ \\
$\mathrm{v}$ & $\mathrm{v}$ & $-\mathrm{u}$ & 1 \\
\hline
\end{tabular}


From Theorem 2.4 it now follows that the algebra $A$ is not von-Neumann finite, and not reversible. Indeed, straightforward calculations shows that

$$
\begin{aligned}
& (1-x-y)(1+x-y)=1, \\
& y(1+x)=0, \\
& (1+x-y)(1-x-y)=1+4 y, \\
& (1+x) y=2 y \text {, }
\end{aligned}
$$

giving explicit counterexamples.

We remark that $B^{\perp} \cap B=\operatorname{Im} B$, so indeed, the algebra $A$ does not satisfy the condition in Theorem 2.2(b) that the norm be non-degenerate on every 3-dimensional subalgebra.

Dropping the condition of flexibility, there exist involutive algebras even with anisotropic norm that are not reversible and not von-Neumann finite.

Example 5.3 Assume that char $F \neq 2$. Let $V$ be a 2-dimensional vector space over $F$, and $q: V \rightarrow F$ a quadratic form such that the form $1 \perp(-q): F \oplus V \rightarrow F,(\alpha, x) \mapsto$ $\alpha^{2}-q(x)$ is anisotropic (e.g., in the real case, $q$ is negative definite). Take an orthogonal basis $(u, v)$ of $V$, and set $u \times v=-v \times u=u$ and $(x, y)=\frac{1}{2}\langle x, y\rangle_{q}=$ $\frac{1}{2}(q(x+y)-q(x)-q(y))$ for all $x, y \in V$. This defines a quadratic algebra structure on $A=F \oplus V$, with $\operatorname{Im} A=V$ and multiplication given by Eq. 2 .

Clearly, $(\cdot, \cdot)$ is symmetric, so $A$ is involutive by Lemma 3.4(a). Moreover,

$$
\mathrm{n}(\alpha, x)=(\alpha, x) \overline{(\alpha, x)}=(\alpha, x)(\alpha,-x)=\alpha^{2}-(x, x)=\alpha^{2}-q(x),
$$

meaning that $\mathrm{n}$ is anisotropic. Now,

$$
\begin{aligned}
& (0, u)(-1, v)=((u, v),-u+u \times v)=0, \\
& (-1, v)(0, u)=((v, u),-u+v \times u)=(0,-2 u) \neq 0,
\end{aligned}
$$

so $A$ is not reversible; and

$$
\begin{aligned}
& (0, u)(-1, u+v)=(0, u)(-1, v)+(0, u)^{2}=-\mathrm{n}(u) \neq 0, \\
& (-1, u+v)(0, u)=(-1, v)(0, u)+(0, u)^{2}=(-\mathrm{n}(u),-2 u) \neq(0, u)(-1, u+v),
\end{aligned}
$$

implying that $A$ is not von-Neumann finite either. It follows from Theorem 2.2(b) that $A$ cannot be flexible, which indeed also can be seen directly from the identities $(u v) u=u^{2}=$ $-u(-u)=-u(v u)$.

For associative algebras, reversibility implies von-Neumann finiteness, and by Theorem 2.4, the same is true for involutive algebras over fields of characteristic different from 2. However, this implication does not hold for general non-associative algebras, as the following example demonstrates.

Example 5.4 Let $A$ be any non-commutative non-quadratic division algebra of dimension 3 over $F$. (Non-quadraticity is automatic if char $F \neq 2$, by [19, Theorem 3].) For example, $A$ may be a twisted field of dimension 3 over a finite field $F$ with at least 3 elements (see [2]).

As $A$ is not quadratic, there exists some element $a \in A$ such that $A=\operatorname{span}\left\{1, a, a^{2}\right\}$, and non-commutativity then implies that $a a^{2} \neq a^{2} a$. Since $A$ is a division algebra, there exists an element $b \in A$ such that $a b=1$. If $b \in \operatorname{span}\{1, a\}$ then $1=a b \in \operatorname{span}\left\{a, a^{2}\right\}$, which is impossible since $1, a, a^{2}$ are linearly independent. Hence $b \notin \operatorname{span}\{1, a\}$ and therefore 
$b a \neq a b$. Thus, $A$ is not von-Neumann finite. But $A$ is reversible, since it is a division algebra.

Funding Open access funding provided by University West.

Open Access This article is licensed under a Creative Commons Attribution 4.0 International License, which permits use, sharing, adaptation, distribution and reproduction in any medium or format, as long as you give appropriate credit to the original author(s) and the source, provide a link to the Creative Commons licence, and indicate if changes were made. The images or other third party material in this article are included in the article's Creative Commons licence, unless indicated otherwise in a credit line to the material. If material is not included in the article's Creative Commons licence and your intended use is not permitted by statutory regulation or exceeds the permitted use, you will need to obtain permission directly from the copyright holder. To view a copy of this licence, visit http://creativecommonshorg/licenses/by/4.0/.

\section{References}

1. Albert, A.A.: Quadratic forms permitting composition. Ann. of Math. (2) 43, 161-177 (1942)

2. Albert, A.A.: Finite noncommutative division algebras. Proc. Amer. Math. Soc. 9, 928-932 (1958)

3. Ara, P., O'Meara, K., Perera, F.: Stable finiteness of group rings in arbitrary characteristic. Adv. Math. 170(2), 224-238 (2002)

4. Cohn, P.M.: Reversible rings. Bull. London Math. Soc. 31(6), 641-648 (1999)

5. Cuenca Mira, J.A., De Los Santos Villodres, R., Kaidi, A., Rochdi, A.: Real quadratic flexible division algebras. Linear Algebra Appl 290, 1-22 (1999)

6. Darpö, E.: Normal forms for the $\mathcal{G}_{2}$-action on the real $7 \times 7$-matrices by conjugation. J Algebra 312(2), 668-688 (2007)

7. Dieterich, E.: Quadratic division algebras revisited. Proc Amer Math Soc 128(11), 3159-3166 (2000)

8. Dieterich, E., Öhman, J.: On the classification of 4-dimensional quadratic algebras over square-ordered fields. J. London Math. Soc. 65(2), 285-302 (2002)

9. Elek, G., Szabó, E.: Sofic groups and direct finiteness. J. Algebra 280(2), 426-434 (2004)

10. Kaplansky, I.: Fields and Rings. Chicago Lectures in Mathematics. University of Chicago Press, Chicago. Reprint of the second (1972) edition (1995)

11. Knus, M.-A., Merkurjev, A., Rost, M., Tignol, J.-P.: The Book of Involutions, Volume 44 of American Mathematical Society Colloquium Publications. American Mathematical Society, Providence (1998)

12. Lam, T.Y.: Introduction to Quadratic Forms over Real Fields, Volume 67 of Graduate Studies in Mathematics. American Mathematical Society, Providence (2005)

13. Lam, T.Y.: Exercises in Modules and Rings. Problem Books in Mathematics. Springer, New York (2007)

14. Lindberg, L.: On the doubling of quadratic algebras. Colloq. Math. 100(1), 119-139 (2004)

15. Lundström, P.: Von Neumann finite endomorphism rings. Indag. Mathem., (N.S.) 14(2), 223-232 (2003)

16. Lundström, P.: Brauer Group Rings. JP J Algebra Number Theory Appl 4(3), 465-494 (2004)

17. McCrimmon, K.: Nonassociative algebras with scalar involution. Pacific J. Math. 116(1), 85-109 (1985)

18. Montgomery, S.: Left and Right Inverses in Group Algebras. Bull. Amer. Math. Soc. 75, 539-540 (1969)

19. Osborn, J.M.: Quadratic division algebras. Trans. Amer. Math. Soc. 105, 202-221 (1962)

20. Passman, D.S.: Idempotents in group rings. Proc. Amer. Math. Soc. 28, 371-374 (1971)

21. Schafer, R.D.: An Introduction to Nonassociative Algebras. Dover publications Inc., New York. Corrected reprint of the 1966 original (1995)

Publisher's Note Springer Nature remains neutral with regard to jurisdictional claims in published maps and institutional affiliations. 